MASTER

CONF -791142--6

ACTINIDE $5 f$ SYSTEMS:

EXPERIMENTAL DETERMINATION OF THE MAGNETIC RESPONSE FUNCTION

by

G. H. Lander

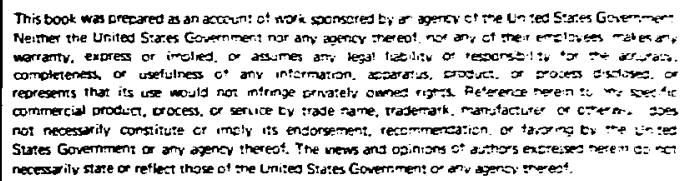

Prepared for

International Conference

on

Crystalline Electric Field

and

Structural Effects on $f$-Electron system

Philadelphia, Pennsylvania

November 12-15, 1979

ARGONNE NATIONAL LABORATORY, ARGONNE, ILLINOIS

Operated under Contract W-31-109-Eng-38 for the U. S. DEPARTMENT OF ENERGY 


\title{
ABSTRACT SHEET
}

\section{ACTINIDE $5 f$ SYSTEMS: EXPERIMENTAL DETERMINATION}

OF THE MAGNETIC RESPONSE FUNCTION*

\author{
G. H. Lander \\ Materials Science Division \\ Argonne National Laboratory \\ Argonne, IL 60439
}

\section{ABSTRACT}

The present state of our understanding of metallic actinide systems is in confusion. Searches for "crystal-field" levels with neutron spectroscopy have, for the most part, been unsuccessful, despite the acknowledged importance of the $5 f$ electrons in determining the magnetic behavior. In systems such as $\mathrm{UAl}_{2}, \mathrm{USn}_{3}$ and UN a broad response function, $S(\vec{Q}, \omega)$, reminiscent of that found in intermediate valent compounds, exists. Neutron inelastic scattering experiments on single crystals have shown the small influence of the crystal field. Instead we find an unusual response function .dominated by the longitudinal susceptibility $x^{2 z}(\vec{Q}, \omega)$ such that transverse excitations -- conventional spin waves -- do not exist at low energies. As yet a detailed theoretical interpretation of the measurements does not exist. Indeed, the small, although not necessaril ${ }_{\bar{y}}$ negligible, role of the crystal field presents conceptual difficulties if we anticipate behavior analogous to that found in mary lanthanide $4 f^{\circ}$ systems. Some alternate approaches will be discussed.

*Work supported by the U. S. Department of Eriergy. 
ACTINIDE $5 f$ SYSTEMS : EXPERIMENTAL DETERMINATION

OF THE MAGNETIC RESPONSE FUNCTION*

\author{
G. H. Lander \\ Materials Science Division \\ Argonne National Laboratory \\ Argonne, IL 60439
}

\title{
INTRODUCTION
}

The advent ten years ago of neutron spectroscopy to identify excited crystal-field (cf) states in lanthanide type materials has greatly added to our understanding of the microscopic interactions in these compounds. 1,2 For example, once the cf scheme is known, one can calculate the magnetization at any field and temperature, compare with experiment, and thus derive exchange terms. ${ }^{3}$ If the material orders then the asy axis, details of the spin-wave $\operatorname{spec}_{-}$ trum, and value of the ordered moment all depend on the cf terms. Indeed, the very presence or absence of magnetic order may depend on whether or not a singlet is the ground state. 5 Despite the difficulty of calculating the cf potential from first principles, its measurement represents a necessary step forward in describing a material. In this respect, one may say it is as necessary as knowing the lattice parameter. In the study of actinide compounds the first step therefore was to determine the cf states.

\section{Early Experiments and Expectations}

The first experiments with neutron spectroscopy were those of Wedgwood ${ }^{6}$ on UX $(X=C, N, P, S$, and Se) and Lander et a1.7 on $\mathrm{USn}_{3}$. Both attempts failed to see any discrete cf levels.

Before proceeding further along this experimental line, let us return to the simple question of whether or not we expect to observe transitions between cf states in actinide materials with 
neutron inelastic spectroscopy. We may first look at investigations of ionic solids with optical techniques. Here the answer is clear, cf states are well defined, as we would expect from the highly correlated $f$ nature of the outer electrons, 8 and the overall energy is roughly a factor of 2 to 5 greater than in the $4 f$ series.9 If we now turn to the metallic compounis, the first study to discuss cf states was by Grunzweig-Genossar et a1.10 They considered the IIX compounds and concluded that the stakle configuration is $5 f^{2}$ with a $\Gamma_{I}$ singlet ground state. The ordering then develops through a strong exchange mixing. Such a model should be immediately verifiable by neutron spectroscopy, especially on compounds diluted with thorium to weaken the exchange interactions. In 1974 Chan and Lam ${ }^{11}$ developed a more complete theory including the effects of $\mathrm{J}$ mixing, but this still assumes well-defined cf levels. Unless the energies between the ground and excited states are always greater than $\sim 12$ THz $\left(\simeq 50 \mathrm{meV} \simeq 400 \mathrm{~cm}^{-1} \simeq 576 \mathrm{~K}\right)$ cf levels should be observable with neutron spectroscopy. For example, by fitting high-temperature susceptibility data Troc and $\mathrm{Lam}^{12}$ proposed that UAS had a $5 \mathrm{f}^{3}$ configuration with a $\Gamma_{8}(1)$ ground state and excited $\Gamma_{6}$ and $\Gamma_{8}(2)$ states at 1.44 and $52.5 \mathrm{THz}$, respectively. The first transition $\Gamma_{8}(1)-\Gamma_{6}$ is exactly in the right region for neutron spectroscopy and evidence for such a transition was reported by Furrer and Murasik ${ }^{13}$ using a polycrystalline sample, but our recent experiments on single crystals show this to be a wrong analysis of the data.

Quite clearly the subject is confused. Our initial (5 years ago) expectation was that in most metallic systems we would find cf levels with neutron spectroscopy. Now we find them hardly at all, and the review I wrnte ${ }^{14}$ in 1976 marks the point at which our naive expectation gave way to reality. Since that time a number of important developments have faken place. First, cf levels have been seen in the U-Pd system, 15,16 thus disspelling the myth that they never can be seen in metallic systems. Second, experiments on $4 f$ systems with intermediate valence have shown that the neutron spectrum consists of a broad Lorentzian response, the energy width of this response being almost independent of temperature. In such systems $\mathrm{cf}$ transitions are rarely seen. Third, experiments on single crystals of $\mathrm{UX}(\mathrm{K}=\mathrm{N}, \mathrm{As}, \mathrm{Sb}, \mathrm{S}$, and $\mathrm{Te})$ have shown that the nature of the response function in these compounds, which all order at relatively high temperatures $(50-250 \mathrm{k})$, is much more complex than in the analogous lanthanide systems.

\section{Neutron Cross Section}

To prepare the discussion for both ordered and nonordered system ve need to consider the fundamental expressions for the thermal neutron cross section, which can be written, 17 


$$
\begin{aligned}
\frac{d^{2} \sigma}{d \Omega d \omega}= & \left\{\left(\frac{r e^{2}}{m_{e} c^{2}}\right)^{2}\left[\frac{1}{2} g f(\vec{Q})\right]^{2} \frac{k_{f}}{k_{1}} \exp [-2 W(\vec{Q})]\right\} \\
& x \sum_{\alpha \beta}\left(\delta_{\alpha \beta}-\hat{Q}_{\alpha} \hat{Q}_{B}\right) \frac{1}{2 \pi t} \int_{-\infty}^{\infty}[\exp (-1 \omega t) \\
& \left.x<\hat{s}_{Q}^{\alpha}(0) \hat{s}_{-Q}^{\beta}(t)>d t\right]
\end{aligned}
$$

We have written down the full expression [Eq. (8.26) in Ref. 18] so as to make some important points.

(i) The section \{\} contains constants, the magnetic form factor $f(\vec{Q})$, and the Debye-Waller factor. We have assumed that the spatial part of the wavefunction is factorizable from the spin operator $\hat{s}_{0}^{\alpha}(t)$ so that the $|\vec{Q}|$ dependence appears in this section only.

(ii) The term $\left(\delta_{\alpha \beta}-\hat{Q}_{\alpha} \hat{Q}_{\beta}\right)$ reminds us that magnetic scattering takes place between components perpendicular to the momentun transfer. This will be important later in ordered systems, although in paramagnets a simple average value of $2 / 3$ enters into the expression.

(iii) The time integral of the spin correlation function enters directly into the cross section.

Two other forms of the cross section are also of importance

$$
\frac{d^{2} \sigma}{d \Omega d \omega}=\{\quad\} \times S(\vec{Q}, \omega)
$$

where

$$
s(\vec{Q}, \omega)=\frac{N}{\pi\left(g \mu_{B}\right)^{2}}[n(\omega)+1] \sum_{\alpha \beta}\left(\delta_{\alpha \beta}-\hat{Q}_{\alpha} \hat{Q}_{\beta}\right) \operatorname{ImX}{ }^{\alpha \beta}(\vec{Q}, \omega)
$$

and through the Kramers-Kronig relationship

$$
\operatorname{ImX}(\vec{Q}, \omega)=\omega \times(\vec{Q}) F(\vec{Q}, \omega)
$$

where the fluctuation spectrum is defined by

$$
\int_{-\infty}^{+\infty} F(\vec{Q}, \omega) d \omega=1 \text {. }
$$


If the spin dynamics are purely relaxational, 1.e., $F(\vec{Q}, t) \sim \exp (-\Gamma(\vec{Q}) \times t)$ then

$$
F(\vec{Q}, \omega)=\frac{1}{\pi} \frac{\Gamma(\vec{Q})}{\Gamma^{2}(\vec{Q})+\omega^{2}} .
$$

The experimental intensity is therefore proportional to the product of $S(\vec{Q}, \omega)$ and a function that takes out the dependence on $|\vec{Q}|$. We may immediately extract $\operatorname{Im\chi }(\vec{Q}, \omega)$, via Eq. (3). Spin waves (or frystal-field excitons) correspond to peaks in the function $\operatorname{Im} \chi(\vec{Q}, \omega)$, which can be expressed as a delta function in one or both of $Q$ and $\omega$. This treatment is not particularly useful in extracting the physics from a spin-wave spectrum of something such as $\mathrm{MnF}_{2}$, but for more complex situations Eq. (3) is a useful form. 18 For paramagnetic systems we can use Eqs. (4) and (5) to see that $S(\vec{Q}, \omega)$ is given by an overall scale factor $x(\vec{Q})$ and a Lorentzian energy distribution, which has a full-width at half maximum (FWHM) of $2 \Gamma(\vec{Q})$.

Non-ordered Systems

Although experiments on $\mathrm{U}_{0.15} \mathrm{Th}_{0.85}$ Se and other diluted $\mathrm{U}$ systems have been performed, 14 no careful data analysis has been presented other than the statement that sharp cf transitions do not exist. The same is true for a very rapid experiment performed some years ago at Brookhaven National Laboratory by $S$. Shapiro and

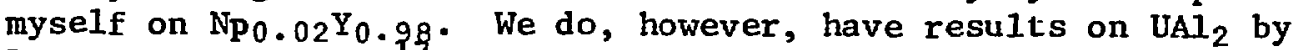
Loewenhaupt et al., 17 who also present qualitative results on $\mathrm{USn}_{3}$ in the same paper.
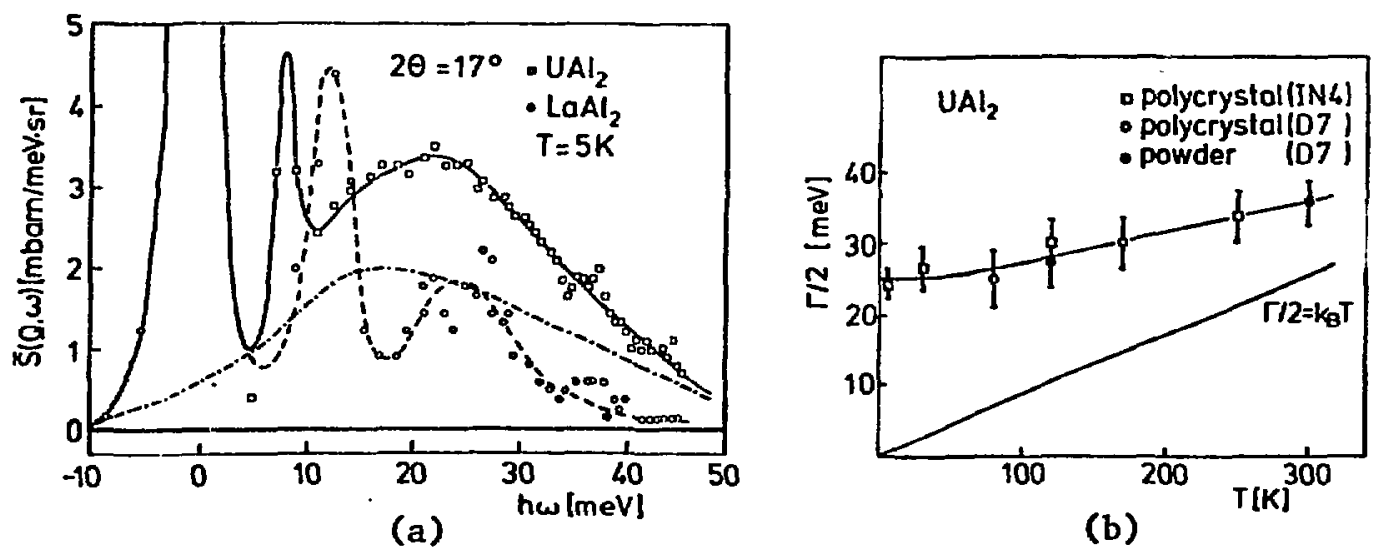

(b)

Fig. 1. (a) Energy loss spectra of $\mathrm{UAl}_{2}$ and $\mathrm{LaAl}_{2}$ at $5 \mathrm{~K}$. Solid line represents $f$ it for $\mathrm{UAl}_{2}$, dashed line for $\mathrm{LaAl}_{2}$. Dashed dotted line shows quasielastic magnetic contribution distorted by detail balance factor $[n(\omega)+1]$. (b) Temperature dependence of half-line width $(4.13 \mathrm{meV}=1 \mathrm{THz})$ in $\mathrm{UA1}_{2}$. (From Loewenhaupt et al., Ref, 17) 
FIgure 1 shows the energy loss spectra (incident energy 51.4 meV $=12.4 \mathrm{THz}$ ) for $\mathrm{UAl}_{2}$ and $\mathrm{LaAl}_{2}$ at $5 \mathrm{~K}$. In addition to the elastic line arising from incoherent nuclear scattering, two inelastic phonon lines are observed. The most important feature is that for $\mathrm{UAl}_{2}$ addicional scattering appears spread over a wide energy range. This additional scattering is well described by Eqs. (2) (5) where the static bulk susceptibility $\chi(\vec{Q}=0)$ is an overall ( $T$ dependent) scale factor and the energy dependence is given by a Lorentzian. The variation of the half width is shown in Fig. 1(b). Such a general magnetic scattering function was found ${ }^{20}$ for the Intermediate valent system ${ }_{1} \mathrm{CePd}_{3}$, and has been found for other nonstable $4 f$ configurations, 18 and forces us at least to consider that many metallic uranium compounds should best be considered in terms of intermediate valence theory.

\section{Ordered Systems}

Studies of ordered systems began with Wedgwood ${ }^{6}$ who noted that the magnetic scattering appeared over a wide energy range and was approximately represented by a gaussian centered at $\omega=0$. These experiments were performed in the paramagnetic state, where most materials show inelastic contributions around $\omega=0$, but the half widths are seldom more than $0.5 \mathrm{THz}, 20$ whereas for the UX materials the half widths were clearly of the order of $3-5 \mathrm{THz}$.

\section{(i) Measurements on UN}

Uranium nitride (NaCl structure, $a_{0}=4.89 \AA$ ) orders antiferromagnetically with the simple type I structure at $53 \mathrm{~K}$. The ordered moment is $0.75 \mu_{B}$ at $0 \mathrm{~K}$. The neutron inelastic scattering experiment failed to find any evidence for discrete excitations. ${ }^{1}$ Instead, in the ordered phase the magnetic response at the (110) reciprocal lattice point, which is the magnetic zone center, is a broad (FWHM $=5 \pm 1 \mathrm{THz}$ ) distribution peaked at $\sim 4 \mathrm{THz}$. One may think of this as a strongly overdamped spin wave with very steep dispersion. Two important points, which will recur in other investigations, is that (a) the response function is primarily longitudinal in nature and (b) the anisotropy gap at the $X$ point [110] is close to that defined by the phonon branches. To understand (a) we must refer back to the orientational factor in Eqs. (1) and (3). In the type I structure the domain symmetry is such that the scattering around [110] and [001] come from a single domain, that with $\mu$ il $[001]$. We may then separate out ${ }^{22}$ the response into transverse $x^{x x}$ (or $x^{y y}$ ) and longitudinal components $x^{z z}$.

Further analysis and experiments are now in progress. Of particular interest will be how the high-temperature broad response function, seen by both Wedgwood ${ }^{6}$ and the Chalk River Group, 21 changes on cooling through $\mathrm{T}_{\mathrm{N}}$. Another question is whether a 
Lorentzian distribution of magnetlc scattering exists even in the ordered phase.

\section{(ii) Measurements on UAs}

Uranium arsenide (NaCl structure, $a_{0}=5.78 \AA$ ) orders with type I antiferromagnetism at $127 \mathrm{~K}$, but has a first order transition to the type IA structure ${ }^{23}$ at $63 \mathrm{~K}$. The ordered moment is $2.2 \mu_{B}$ at $5 \mathrm{~K}$. Neutron inelastic scattering experiments have only just been completed, 24 so that the results are very preliminary. Nevertheless, some simple statements are possible. First, no collective excitation has been observed in the ordered state. Second, the scattering may be characterized by a scale factor $\chi(\vec{Q})$ and a Lorentzian energy spectrum centered about $\omega=0$ in the ordered state.

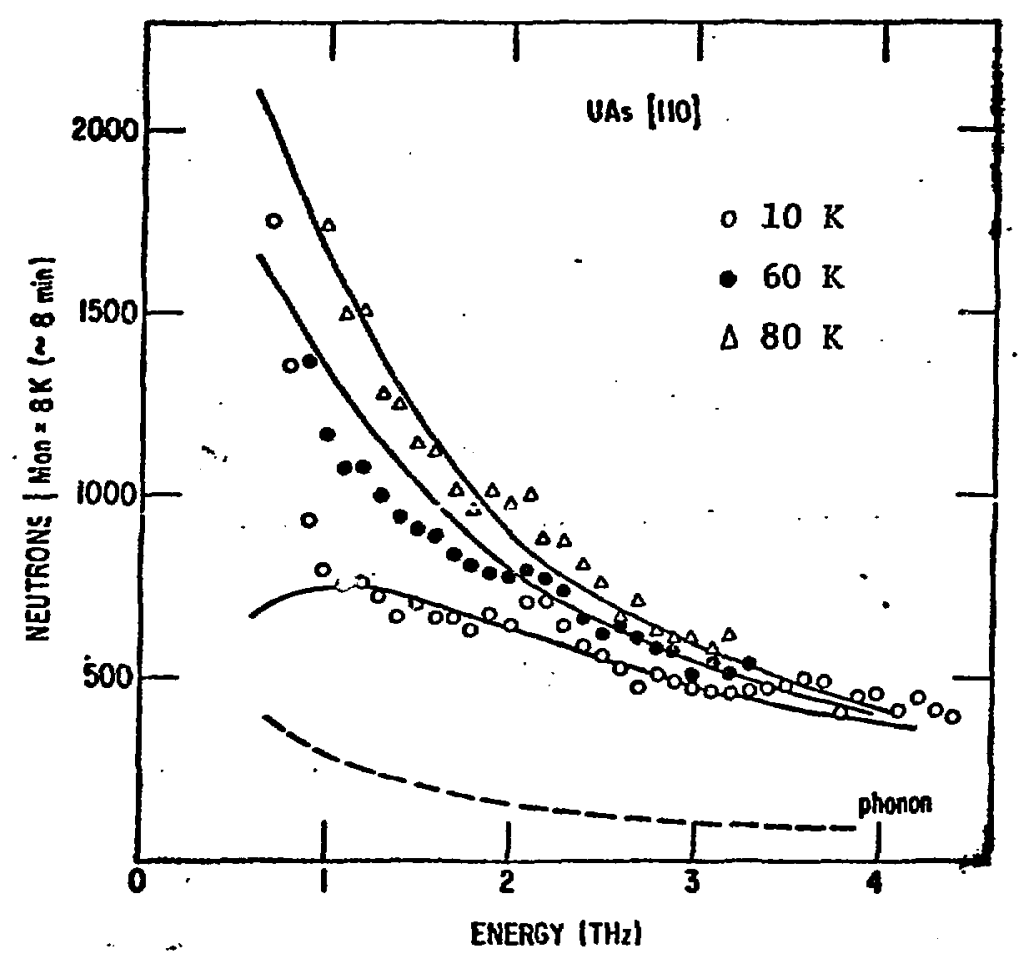

Fig. 2. Experimental scans $\left(k_{f}=3.6 \AA^{-1}\right)$ from a single crystal of UAs at different temperatures. The phonon contrihution (shown for $T=80 \mathrm{~K}$ ) is obtained from the $Q^{2}$ dependence of the scattering. The lines represent the variation in scattering expected if $\chi(110)=700$ and $\Gamma(110)=1.1 \mathrm{THz}$. The change in shape is simply a feature of the thermal population and detail balance factor. We have not subtracted the tail of the incoherent scattering, hence the upturn in experimental intensity for $\omega<1 \mathrm{THz}$ and $\mathrm{T}=$ $10 \mathrm{~K}$ is not significant. 
We show in Fig. 2 scattering from UAs. To fit the data we have separated the nuclear and magnetic parts by the $Q$ dependence (for example, at each temperature scans were done at both the [110] and [330] zone centers) and then usẹd the expression derived from Eqs. (3) - (5). Similar curves for $\vec{Q}=[001]$ are shown in Fig. 3.

Without going into great length, we can note the following:

(a) The magnetic scattering is very strong. The incoherent phonon contribution is sma11 $(<20 \%)$, as also is the instrumental background of 440 counts on this scale, and which has not yet been subtracted. (b) Since $x(\vec{Q}=[001])=2 x^{x x}$ and $x(\vec{Q}=[100])=x^{x x}+x^{22}$, then we can roughly deduce that $x^{z z} \approx 2 \frac{1}{2} x^{x x}$.

(c) Above $\mathrm{T}_{\mathrm{N}}(=127 \mathrm{~K})$ the spectra (not shown) indicate a drop in $x(\vec{Q})$ but rather little change in $\Gamma(\vec{Q})$. This is exactly what is expected if we believe the response function is a measure of the fluctuation time, and therefore insensitive to ordering, whereas the staggered susceptibility $\times(\vec{Q})$ must be greater in the ordered stata. (d) In the [110] scans the collective excitation possibly begins to coalesce at $\sim 2.1 \mathrm{THz}$ (this srall peak has been also seen with $k_{f}=3.36 \mathrm{~A}^{-1}$ ) but line-broadening effects prevent its observation away from this point.

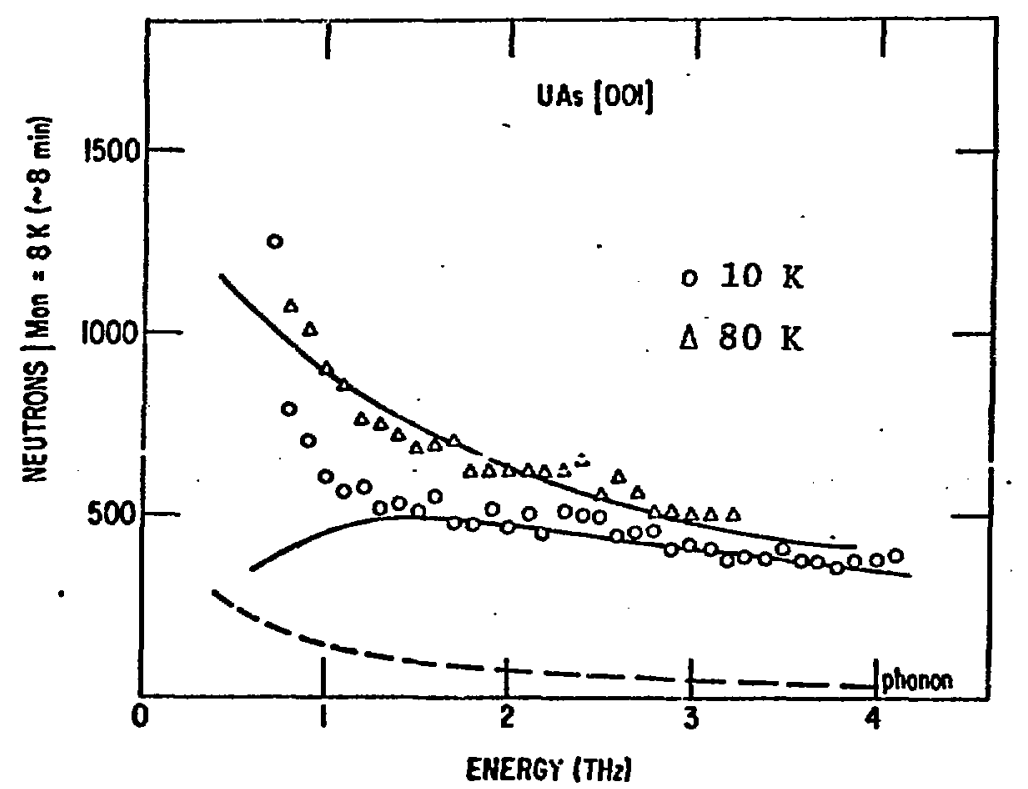

Fig. 3. Experimental scans $\left(k_{f}=3.6 \AA^{-1}\right)$ and preliminary fits at the [001] point in UAs. The solid curves are as in Fig. 2 except that $\chi(001)=400$ and $\Gamma(001)=1.6 \mathrm{THz}$. The phonon function, again shown for $T=80 \mathrm{~K}$, is the same as in Fig. 2. . 


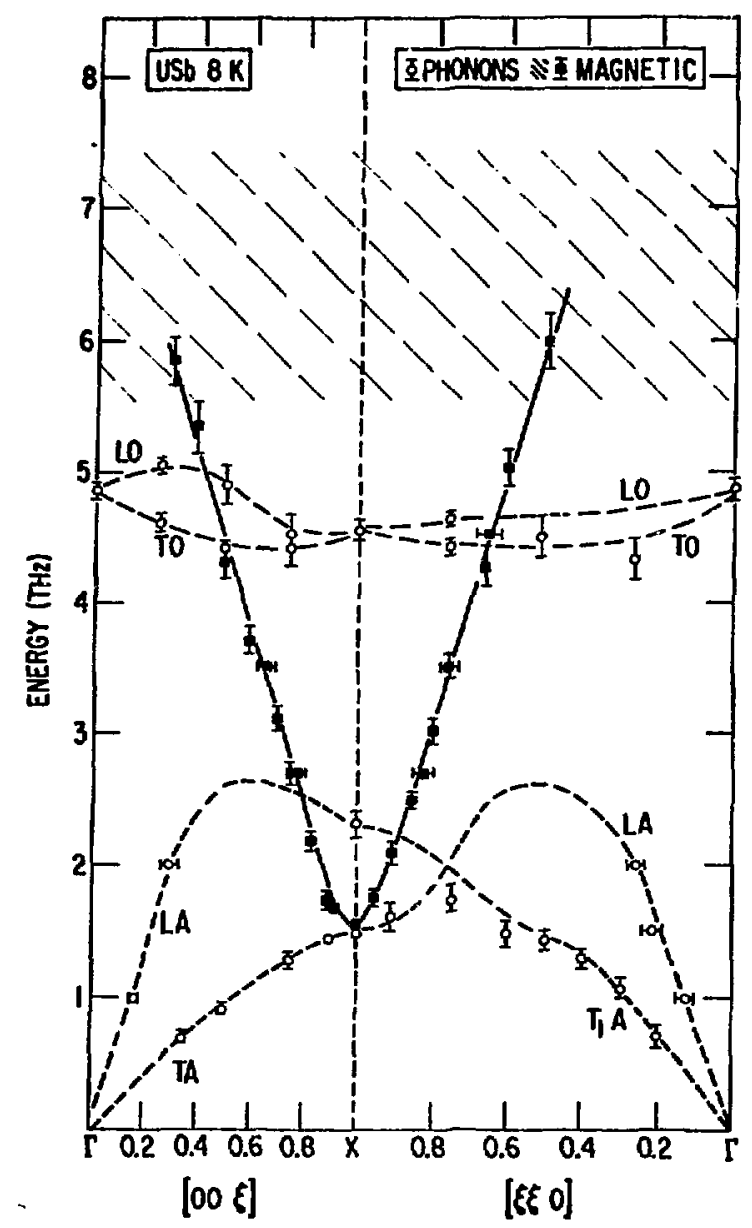

Fig. 4. The dispersion curves for USb; energy plotted against wave-vector transfer $\bar{Q}$ (in units of $2 \pi / a)$. The dashed lines represent the phonons. The magnetic modes are represented by solid squares (the collective excitation) and the hatched area (excitonic level).

\section{(iii) Measurements on USb}

Uranium antimonide (NaCl structure, $a_{1}=6.20 \AA$ ) orders with the type I structure at $240 \mathrm{~K}$. The ordered moment is $2.8 \mu_{\mathrm{B}}$ at $0 \mathrm{~K}$. In contrast to the rather featurelesi spectra discussed above for UN and UAs, the excitation spectra for USb has a number of most intriguing features. 25

The dispersion curves are shown in Fig. 4. The most important point of the USb experiment concerns the observation of a longitudinally polarized magnetic excitation, centered at the X-point, with steeply rising branches. As the temperature is raised the intensity of this collective mode decreases so that by $\mathrm{T}_{\mathbb{N}} / 2$ it is unobservable. Second, we see a dispersionless exciton (cf level) at $\sim 6 \frac{1}{2} \mathrm{THz}$. Third, we observe a broad response function which is centered on zero energy and (experimentally) appears to increase in intensity as the temperature is raised. 
Let us examine these in the context of the UN and UAs experiments. The broad response function is clearly similar to that shown in Figs. 2 and 3 and experimentally in USb we also finü a much stronger signal at [110] than [001]. The of level in USb at $\sim 6 \frac{1}{2}_{2} \mathrm{THz}$ is in excellent agreement with predictions based on formfactor measurements, ${ }^{26}$ so that this can be reasonably assigned to a transition between states of different symmetry. If the splitting $\Delta \mathrm{E}$ is proportional to $\mathrm{a}_{\mathrm{o}}{ }^{-5}$, as expected from of considerations, then $\Delta \mathrm{E} \sim 9 \mathrm{THz}$ in UAs and $\sim 21 \mathrm{THz}$ in $\mathrm{UN}$. Both values are rather large for neutron spectroscopy and might well be missed, especially as the matrix element in USb is small. Finally, we come to the collective longitudinal mode. Where has this gone in UN and UAs? We cannot, of course, answer this question unambiguously. It seems highly probably though that the response is strongly overdamped in UN and UAs, but would like to appear at approximately the acoustic phonon X-point frequency, which is what happens in USb (see Fig. 4). The most plausible suggestion is that the strong indirect $5 f-5 f$ interaction through the anions and/or conduction electrons leads to suppression of the longitudinal fluctuation. We might expect this to be a function of lattice parameter, and its absence in those materials with small $\mathrm{U}$ separation is at least consistent with this. view.

\section{CONCLUSIONS}

We have discussed at some length the neutron cross section to draw attention to the fact that more than just cf levels can be seen with neutron spectroscopy. Progress since $1976^{14}$ has been substantial -- at least from an experimental view, but it is also clear that we have a long way to go before we can claim an understanding. We have shown that the broad magnetic response function seen in intermediate valence $4 f$ compounds is also present in many $U$ compounds, independent of whether they order or not. In one material, USb, an unusual longitudinal excitation has been observed. This corresponds to a correlated fluctuation in the magnitude of $\left\langle\mathrm{J}_{z}\right\rangle$, and one might therefore speculate that it is a collective valence fluctuation, although such a highly provocative statement is not yet supported by quantitative theory. The conventional spin-wave (transverse) response in these materials is clearly at very high energies, as a consequence of the uniaxial anisotropy, 22 and has not been seen with neutron experiments. The inability of the neutrons to see the longitudinal mode in UN and UAs is probably because of lifetime broadening effects, arising from interaction with the conduction electrons, or a widening of the $5 f$ band width if we choose to consider a band structure approach.

In this paper I have suggested that the difficulties and challenge of neutron spectroscopy in $U$ compounds is because many of them show characteristics of intermediate valence. Since the 
two ground states, $5 f^{2}$ and $5 f^{3}$, may both support magnetic ordering, these systems may well turn out to be as interesting as TmSe, a compound of much current interest. The next few years will show to what extent these suggestions are valid.

\section{ACKNOWLEDGMENTS}

Discussions with W. J. L. Buyers, B. R. Cooper, T. M. Holden, S. K. Sinha, and W. G. Stirling are much appreciated. These colleagues should not be held responsible for the possibly controversial views expressed here! Finally, the cooperation, and continued enthusiasm of Oscar Vogt of ETH, Zurich in growing the crystals involved in these studies is of immeasurable importance.

\section{REFERENCES}

1. K. C. Turberfield, L. Passell, R. J. Birgeneau, and E. Bucher J. Appl. Physics 42, 1746 (1971).

2. See many papers in "Crystal Field Effects in Metals and Alloys," A. Furrer, ed., Plenum Press, New York City (1977).

3. An example is T. O. Brun, J. S. Kouvel, and G. H. Lander, Phys. Rev. B 13, 5007 (1976).

4. T. M. Holden, E. C. Sven ion, W. J. L. Buyers, and O. Vogt,

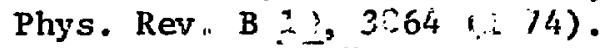

5. B. R. Cooper in "Magnetic Properties of Rare Earth Metals",

R. J. E11iott, ed., P1Enum Press, New York City (1972).

6. F. A. Wedgwood, J. Phys. C 7, 3203 (1974).

7. G. H. Lander, T. O. Rrun, B. W. Veail, and D. J. Lain, in 'Proc. of Conf. on Crystalline Electric Field Effects in Mecals and Alloys", R. A. Devine, ed., University of Montreal (1974) p. 480.

8. B. G. Wybourne, "Spectroscopic Properties of Rare Earths", Interscience, New York (1965) p. 198 et seq.

9. W. T. Carnall, H. M. Crosswhite, H. Crosswhite, J. P. Hessler,

C. Aderhold, J. A. Caird, A. Paszek, and F. W. Wagner, "Proc.

of 2nd Int. Conf. on the Electronic Structure of the Actinides", J. Mulak, W. Suski, and R. Troc, eds., Polish Academy of Sciences (1977) p. 105. See also M. E. Hendricks, et al., J. Chem. Phys. 60, 2095 (1974) and W. T. Carna11, et a1., J. Chem. Phys. 61, 4993 (1974).

10.J. Grunzweig-Genossar, Phys. Rev. 173, 562 (1968).

11.S. K. Chan and D. J. Lam in "The Actinides: E'lectronic Structure and Related Properties", A. J. Freeman and J. B. Darby, Academic Press, New York (1974), Vol. I. Ch. 1.

12.R. Troc and D. J. Lam, Phys. Stat. Sol. 65 b, 317 (1974).

13.A. Furrer and A. Murasik, Helvetica Physica Acta 50, 447 (1977).

14.G. H. Lander, in "Crystal Field Effects in Metals and Alloys",

A. Furrer, ed., Plenum Press, New York City (1977), p. 213.

15.N. Shamir, M. Melamud, H. Shaked, and M. Weger, Physica 94B, 225 (1978). 
16.A. F. Murray and W. J. L. Buyers, see Proceedings of this Conference.

17.W. Marshall and S. Lovesey, "Theory of Thermal Neutron Scattering", Oxford, Clarendon Press. (1971) ch. 8.

18.S. M. Shapiro, J. D. Axe, R. J. Birgeneau, J. M. Lawrence, and R. D. Parks, Phys. Rev. B 16, 2225 (1977).

19.M. Loewenhaupt, S. Horn, F. Steglich, E. Holland-Moritz, and G. H. Lander, J. de Fhysique 40, C4-142 (1979) .

20.E. Holland-Moritz, M. Loewenhaupt, W. Schmatz, and D. K. Wohlleben, Phys. Rev. Letters 38, 983 (1977).

21.W. J. L. Buyers, T. M. Holden, E. C. Svensson, and G. H. Lander, in "Proc. of the International Symposium on Neutron Ineiastic Scattering", IAEA, Vienna, (1978) p. 239. T. M. Holden, et al., J. de Physique 40, C4-31 (1979) (abstract only).

22.G. H. Lander, S. K. Sinha, D. M. Sparlin, and 0. Vogt, Phys. Rev. Letters 40,523 (1978) and references therein.

23.G. H. Lander, $\vec{M}$. H. Mueller, and J. F. Reddy, Phys. Rev. B 6 , 1880 (1972).

24.G. H. Lander, W. G. Stirling, and O. Vogt, Experiment at Inst. Laue Langevin, August/Sept. 1979. Unpublished.

25.G. H. Lander, W. G. Stirling, and 0. Vogt, Phys. Rev: Letters 42, 260 (1979); and Phys. Rev. B (1980) in press.

26.G. H. Lander, M. H. Mueller, D. M. SFarlin, and O. Vogt, Phys. Rev. B 14, 5035 (1.976). 